\title{
A molecular perspective on water vapour accommodation into ice and its dependence on temperature
}

\author{
Daniel Schlesinger ${ }^{1,2}$, Samuel J. Lowe ${ }^{1,2}$, Tinja Olenius ${ }^{1,2,+}$, Xiangrui Kong $^{3}$, Jan B. C. \\ Pettersson $^{3}$ and Ilona Riipinen ${ }^{1,2}$ \\ ${ }^{1}$ Department of Environmental Science (ACES), Stockholm University, Svante Arrhenius väg 8, SE-106 91 \\ Stockholm, Sweden \\ ${ }^{2}$ Bolin Centre for Climate Research, Stockholm University, SE-106 91 Stockholm, Sweden \\ ${ }^{3}$ Department of Chemistry and Molecular Biology, Atmospheric Science, University of Gothenburg, Kemigården \\ 4, SE-412 96 Gothenburg, Sweden \\ ${ }^{\dagger}$ Current address: Swedish Meteorological and Hydrological Institute (SMHI), Research Department, Air Quality \\ Research Unit, SE-601 76 Norrköping, Sweden
}

\begin{abstract}
Accommodation of vapour phase water molecules into ice crystal surfaces is a fundamental process controlling atmospheric ice crystal growth. Experimental studies investigating the accommodation process with various different techniques report widely spread values of the water accommodation coefficient on ice, $\alpha_{\text {ice, }}$ and the results on its potential temperaturedependence are inconclusive. We run molecular dynamics simulations of molecules condensing onto the basal plane of ice Ih using the TIP4P/Ice empirical force field and characterize the accommodated state from this molecular perspective, utilizing the interaction energy, the tetrahedrality order parameter and the distance below the instantaneous interface as criteria. Changes of the order parameter turn out to be a suitable measure to distinguish between surface and bulk states of a molecule condensing onto the disordered interface. In light of the findings from the molecular dynamics, we discuss and reanalyse a recent experimental data set on $\alpha_{i c e}$ obtained with an environmental molecular beam (EMB) setup [Kong et al, Journal of Physical Chemistry A, 2014] using kinetic molecular flux modelling, aiming at a more comprehensive picture of the accommodation process from a molecular perspective. These results indicate that the experimental observations indeed cannot be explained by evaporation alone. At the same time our results raise the issue of rapidly growing relaxation times upon decreasing temperature, challenging future experimental efforts to cover relevant time scales. Finally, we discuss the relevance of the water accommodation coefficient on ice in the context of atmospheric cloud particle growth processes.
\end{abstract}




\section{Introduction}

Condensation and deposition of water vapour onto liquid water and ice surfaces is a key process in the Earth's atmosphere, driving e.g. the growth of cloud droplets and ice crystals and hence influencing the evolution and properties of clouds (Lamb and Verlinde, 2011; Pruppacher and Klett, 2010). The key parameter governing the thermodynamic equilibria between water vapour and its various condensed phases is the saturation vapour pressure above the liquid or solid phase, which is directly linked to the evaporation rate of water molecules from the condensed phase (Lamb and Verlinde, 2011; Pruppacher and Klett, 2010; Seinfeld and Pandis, 2006). The difference between the ambient water vapour concentration and the equilibrium vapour pressure determines to a large degree the net growth or evaporation of water hydrometeors (Lamb and Verlinde, 2011; Pruppacher and Klett, 2010). An additional coefficient known as the mass accommodation coefficient $\alpha$ (also sometimes called the condensation or evaporation coefficient) has been proposed to modulate the condensation and evaporation fluxes at the free molecular regime (Fuchs and Sutugin, 1970; Seinfeld and Pandis, 2016). The most common formulation defines $\alpha$ as the fraction of incoming molecules, as determined by kinetic gas theory, that stick and accommodate into the bulk condensed phase at the absence of evaporation, or in reverse, the ratio between the evaporation rate and the maximum kinetic evaporation into vacuum (i.e. at the absence of condensation or deposition) (Kolb et al., 2010; Seinfeld and Pandis, 2016). Being a primarily kinetic parameter, the effect of $\alpha$ disappears at the limit of the continuum regime where the condensation or deposition becomes diffusion-limited, and can thus be described by the macroscopic transport equations (see e.g. (Vehkamäki and Riipinen, 2012) and references therein). In the literature investigating water condensation, deposition and evaporation, however, the definition and physical interpretation of the mass accommodation coefficient vary. Most studies relate $\alpha$ to an additional energetic barrier related to the restructuring of the surface as it takes up a molecule (Varilly and Chandler, 2013; Vehkamäki and Riipinen, 2012). For ice surfaces, the mass accommodation coefficient is alternatively related to a reorientation of the condensing molecule (Lohmann et al., 2016). Some studies describe particle phase transport through an effective mass accommodation coefficient (e.g. (Sinha et al., 2018), (Liu et al., 2019)). The uptake coefficient, often denoted with $\gamma$, can be used from 
the free molecular to the continuum regimes, and it converges with $\alpha$ in the kinetic regime (Kulmala and Wagner, 2001). The ambiguity in the definition of $\alpha$ makes it difficult to compare and interpret experimental data, and eventually resolve the value of $\alpha$ that should be used in e.g. cloud models (Hienola et al., 2001). Furthermore, it is not fully clear in which conditions the accommodation process can be described by a single accommodation parameter $\alpha$, instead of treating it as a two-step process governed by separate surface and the bulk accommodation coefficients $\alpha_{\mathrm{s}}$ and $\alpha_{\mathrm{b}}$ (Julin et al., 2013; Pöschl et al., 2007). Such a description is particularly relevant for non-homogeneous, multi-layered surfaces such as ice, in which a disordered interface (Bartels-Rausch et al., 2014) separates the crystalline ice from the vapour phase (Neshyba et al., 2009a).

For liquid water, numerous experimental and theoretical studies of $\alpha$ find values close to unity (e.g. Miles et al., 2012; Winkler et al., 2004, 2006) while others find significantly lower values (see, e.g., Kolb et al. 2010). Accurately constraining $\alpha$ has proven challenging with standard experimental techniques due to e.g. uncertainty in other key parameters needed for the interpretation of the data, especially evaporation rates, diffusion coefficients and the ambient water vapour concentration and temperature (Miles et al., 2012). Julin et al. (2013) studied the condensation and evaporation of water with a combination of MD, kinetic modelling and experimental data at $T=268-300 \mathrm{~K}$, and concluded that their results were in line with $\alpha>0.99$ for both planar as well as curved surfaces. Furthermore, it was concluded that simple kinetic modelling representing the accommodation process without explicitly accounting for the surface phase (i.e. as a one-step process with a single $\alpha$ ) was sufficient. In the case of ice, however, accommodation involves the disordered interface. Close to the melting temperature $T_{\mathrm{m}}$, this interface is often referred to as the "pre-melting layer" or the "quasi-liquid layer", and its thickness and properties strongly depend on temperature (Dash et al., 2006; Slater and Michaelides, 2019). Upon cooling below $T_{\mathrm{m}}$, the thickness quickly drops to molecular dimensions and at temperatures far below $T_{\mathrm{m}}$, i.e. $T_{\mathrm{m}}-T<\approx 25 \mathrm{~K}$, only the topmost molecular layer is affected by entropic disordering (Conde et al., 2008; Limmer and Chandler, 2014). Here we investigate the role of this disordered layer in the accommodation process, treating the accommodation process to the surface vs. uptake to the bulk crystal as separate processes manifested in different $\alpha_{\mathrm{s}}$ and $\alpha_{\mathrm{b}}$, respectively. 
A wide range of experimental techniques has been applied in the past decades for probing the condensation and evaporation of water vapour on and from ice, and extracting an accommodation coefficient. These techniques include various molecular beam-based approaches (Brown et al., 1996; Bryson et al., 1974; Kong et al., 2014; Koros et al., 1966), FTIR spectroscopy (Tolbert and Middlebrook, 1990), optical interference (Brown et al., 1996; Haynes et al., 1992), use of electrodynamic balance in controlled vapour concentrations (Magee et al., 2006), microbalances and/or mass spectrometric techniques coupled to different flow reactor, vacuum chamber or Knudsen cell designs (Kramers and Stemerding 1951; Leu 1988; Chaix, Allanic, and Rossi 2000; Fluckiger and Rossi 2003; C. Delval, Fluckiger, and Rossi 2003; Delval and Rossi 2005; Pratte, van den Bergh, and Rossi 2006) as well as monitoring the evolution of aerosol particles and hydrometeors in flow tubes or condensation/evaporation chambers (Choularton and Latham, 1977; Isono and Iwai, 1969; Skrotzki et al., 2013). In general, the techniques applied for assessing the accommodation coefficient at higher temperatures tend to report on average lower values than the approaches focusing on lower temperatures (see e.g. Kong et al. 2014). A decreasing temperature trend using the same technique throughout the temperature range is reported by e.g. Delval et al. (2003) Pratte et al. (2006) and Kong et al. 2014 for $120-240 \mathrm{~K}$. On the other hand, Magee et al. (2006) and Choularton and Latham (1977) report an increasing trend with temperature at 190-260 K. Some studies, like Kramers and Stemerding (1951) for 213 233 K, Haynes et al. (1992) for 20 - 185 K, and Skrotzki et al. (2013) for 190 and 235 K, report values close to unity with practically no temperature-dependence at all. The differences between the values obtained by different measurement approaches can result from uncertainties in the measurable quantities or e.g. potential measurement artefacts. However, the ambiguities in the mere definition of the accommodation coefficient might play a role in the variation of the observation results. As the definition is built into the models that are used to link the directly measurable quantities to the underlying physical processes, variations in the approach for interpreting the measurements may contribute to the deviations in the values deduced for $\alpha$.

Molecular dynamics (MD) simulations are a useful tool for addressing surface processes, and particularly valuable for identifying the molecular and structural properties manifesting themselves as an accommodation coefficient (see e.g. Julin et al. 2013). While such simulations are challenging to perform for ice at low temperatures, since the relaxation 
times are long compared to feasible simulation times, they are applicable for the ice interface, where the characteristic time scales lie between those of the corresponding supercooled liquid and the solid. MD simulations have been applied by Neshyba et al. (2009) to study the adsorption and bulk accommodation processes of water molecules on an ice (0001) surface involving the disordered layer, reporting values for the surface accommodation coefficient of $\alpha_{\mathrm{s}}>0.99$ and the bulk accommodation coefficient of $\alpha_{\mathrm{b}}=0.6$ at $T=250 \mathrm{~K}$ using a 6 -site water model. These authors define the surface accommodation coefficient as the fraction of condensing molecules sticking to the surface while the bulk accommodation coefficient was calculated from the ratio of transitions between the quasi-liquid layer into the bulk layer, and transitions between the quasi-liquid layer into bulk and vapour phase. The authors emphasise, however, that this result depends on the choice of the layer in which the accommodation is assumed to take place. In a more recent study, MD simulations were used to inspire a continuum description of ice crystal growth dynamics which takes the quasi-liquid and faceted structure of the ice surface into account (Neshyba et al., 2016). Mohandesi and Kusalik focused on the ice growth rate from the vapour phase as obtained from MD simulations of the TIP4P/2005 model of water (Mohandesi and Kusalik, 2018), obtaining the temperaturedependent ice growth rate with maxima of $7 \mathrm{~cm} \mathrm{~s}^{-1}$ for the prism face and $4 \mathrm{~cm} \mathrm{~s}^{-1}$ for the basal face, respectively. The latter study did not attempt to obtain a bulk accommodation coefficient although information about it must be contained in the data.

In this paper we use a combination of MD simulations and kinetic flux calculations to develop a molecular definition of $\alpha$ for water vapour over ice over a wide temperature range, exploring the influence of the disordered interface on the accommodation process. First, we study the properties of bulk ice as compared with the surface and vapour phases to establish criteria for when a molecule can be considered to be accommodated as part of the surface or the bulk ice. Second, we follow an ensemble of trajectories of molecules originating from the gas phase as they penetrate into the condensed phase, comparing the time evolution of their energetics and orientation to the criteria established for surface and bulk accommodation. Finally, we explore potential uncertainties in interpreting experimental data on accommodation through a case study of analysing the data from Kong et al. 2014 with a kinetic flux modelling approach using insights from the molecular simulations. 


\section{Methods}

\subsection{Molecular dynamics simulations}

Here we focus on the accommodation process of single molecules condensing onto ice surfaces at different temperatures. We use the program package GROMACS ver. 5.1.4 (Abraham et al., 2015) and the TIP4P/Ice model of water (Abascal et al., 2005) to simulate systems initially containing a slab of ice ( $N=432$ molecules) and one gas phase molecule (Fig. 1a) at temperatures of $T=100,170,200,230$ and $250 \mathrm{~K}$, the melting temperature of TIP4P/Ice being $T_{\mathrm{m}}=272.2 \mathrm{~K}$ (Abascal et al., 2005). The ice slabs are equilibrated prior to the production runs for $t_{\text {eq }}=100 \mathrm{~ns}(T=100 \mathrm{~K}, 170 \mathrm{~K}$ and $200 \mathrm{~K})$ and $t_{\mathrm{eq}}=10 \mathrm{~ns}(T=230 \mathrm{~K}$ and $250 \mathrm{~K})$. After the initial equilibration in the isothermal-isobaric (NPT) ensemble using the Parrinello-Rahman barostat (Parrinello and Rahman, 1981), 100 frames are extracted from the trajectories at each temperature.

For each of the 100 configurations we add a gas phase molecule approximately $\Delta z \approx 2$ $\mathrm{nm}$ above the surface with velocities initialised according to the Maxwell-Boltzmann distribution at the respective temperature $T$ and switch to the canonical (NVT) ensemble. All molecules, excluding the vapour phase molecule, are coupled to a heat bath using the Bussi thermostat (Bussi et al., 2007) and propagated in time at a time step of $d t=2 \mathrm{fs}$ for total simulation times of $10 \mathrm{~ns}(T=200,170,100 \mathrm{~K}), 2 \mathrm{~ns}(T=230 \mathrm{~K})$, and $1 \mathrm{~ns}(T=250 \mathrm{~K})$. After velocity initialization, the condensing molecule is not coupled to the thermostat at any time. Coulomb interactions are treated with the particle-mesh Ewald method (see, e.g. (Frenkel and Smit, 2001). Both van der Waals and real-space Coulomb interactions are cut-off at $0.85 \mathrm{~nm}$. We use three different indicators for characterising the accommodation process: the distance to the instantaneous interface, the short-range interaction energy, and an order parameter. 


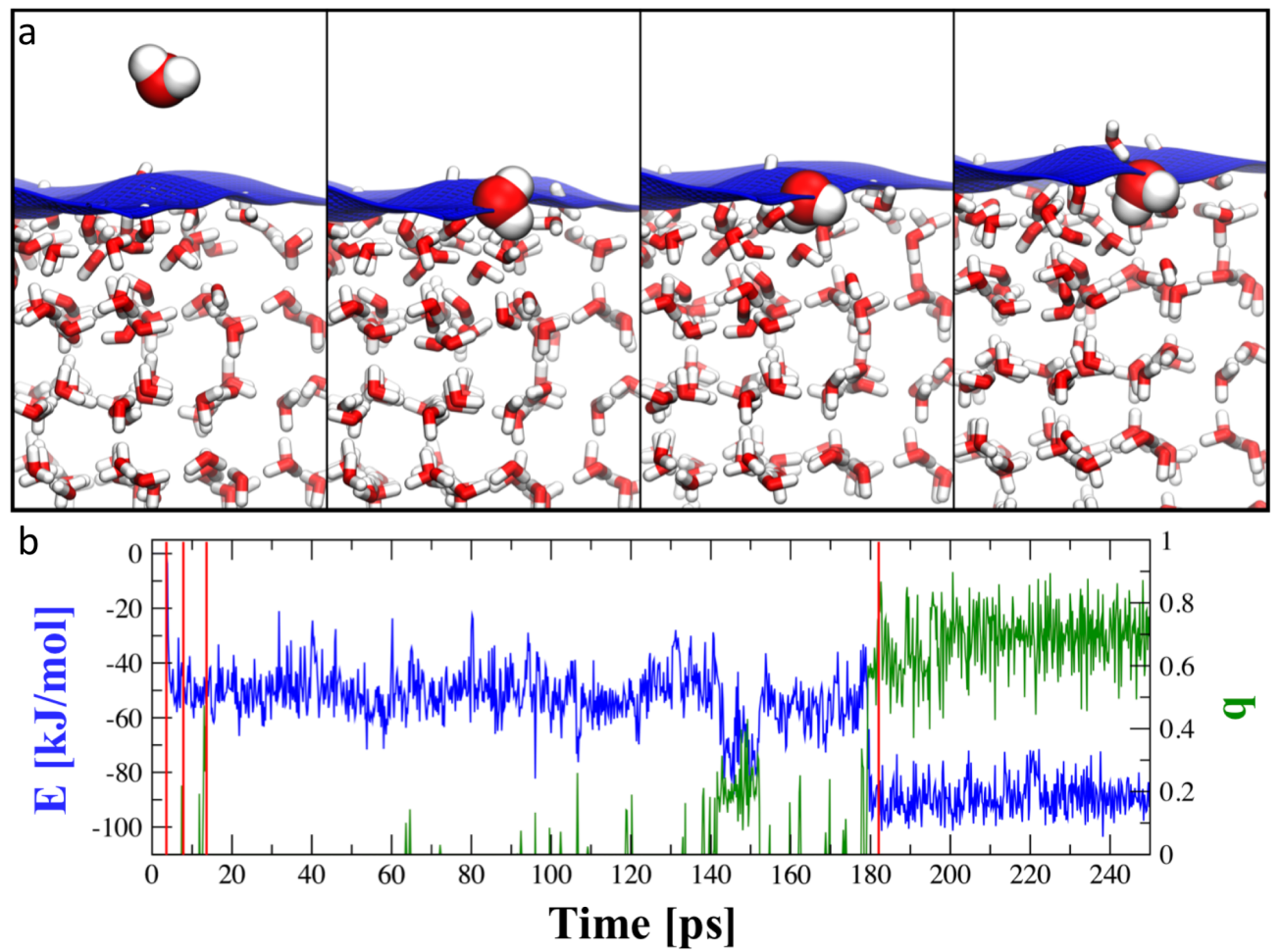

Fig. 1: (a) Snapshots from a simulation of a condensing molecule on an ice surface at $T=200$ $\mathrm{K}$ at simulation times $t=3.4,7.6,13.4$, and $182 \mathrm{ps}$ (left to right). We use a different representation for the condensing molecule than for bulk molecules for clarity. The instantaneous interface indicated in blue is computed using the procedure described in the Methods section. The snapshots were rendered with VMD (Humphrey et al., 1996). (b) Shortrange interaction energy $E$ (left vertical axis, blue line) and tetrahedrality parameter $q$ (right vertical axis, green line) from the first $250 \mathrm{ps}$ of a simulation at $T=200 \mathrm{~K}$ as functions of time. The times at which the snapshots of panel (a) are taken are indicated with red vertical lines at $t=3.4,7.6,13.4$, and $182 \mathrm{ps}$.

\subsubsection{Definition of the surface}

The instantaneous surface of the ice slab is defined as the half bulk-density iso-surface of the density field obtained by adding contributions from Gaussians, centred around each oxygen atom, on a $50 \times 50 \times 50$ points $3-D$ grid following the method detailed in Ref. (Willard and Chandler, 2010). This definition allows us to measure the distance of the condensing molecule to the surface which is rough on a molecular level, and to plot other characterizing quantities, such as the order parameter, as a function of the distance. The condensing molecule is omitted from the determination of the surface and can thus appear to locally deform the interface. The result of this analysis is illustrated in Fig. 1a. While this method was originally introduced for liquid interfaces, it is used here for ice surfaces as it is a robust method regardless of the degree of disorder in the interface. 


\subsubsection{Definition of the order parameter}

The order parameter characterizing the orientation of molecule $i$ with respect to the surrounding molecules is here defined as the tetrahedrality parameter

$$
q_{i}=1-\frac{3}{8} \sum_{j}^{3} \sum_{k=j+1}^{4}\left(\cos \psi_{j k}+\frac{1}{3}\right)^{2}
$$

similar to the definition in Ref (Errington and Debenedetti, 2001) (see also Fig. 1b for an example), but without thermal averaging. $\psi_{j k}$ denote the angles between the lines connecting the oxygen atoms of the central molecule $i$ with those of its nearest neighbors $j$ and $k$. The original order parameter from Ref (Errington and Debenedetti, 2001) is unity for perfectly tetrahedral configurations, i.e. an ideal ice lattice, and vanishes for random configurations. In our case, without thermal averaging, $q_{i} \in[-3,1]$, but the value $q_{i} \approx 1$ for tetrahedral configurations still holds. Therefore, for molecules occupying undisturbed ice lattice sites, $q_{i}=1$, while we define $q_{i}:=0$ for molecules with less than four neighbors within a cut-off distance of $d_{\text {cut }}=3.7 \AA$. We only consider values of $0 \leq q_{i} \leq 1$ and omit possible negative values since the corresponding highly distorted local $\mathrm{H}$-bond structures are not of interest in the current context. We will refer to the order parameter as "tetrahedrality parameter" $q$.

\subsubsection{Definition of the short-range interaction energy}

The transition of a molecule from the vapour to the condensed phase can be characterized by the intermolecular interaction energy. During the transition, the interaction energy changes from no, or weak, interactions to strong attractive interactions. Here, we use the short-range (SR) interaction energy, i.e. the sum of Coulomb and van der Waals interactions, of a condensing molecule with all other molecules within the cut-off radius of $0.85 \mathrm{~nm}$ (see simulation detail above, and Fig. 1b for an example) and refer to it simply as "interaction energy" $E$.

\subsection{Re-analysis of environmental molecular beam data using molecular flux modelling}


To complement the molecular perspective rising from the MD simulations and discuss it in the context of observables linked to accommodation, we use molecular flux modelling based on thermodynamic and kinetic theories to re-analyse relatively recent experimental data on the accommodation coefficient of water into a bulk ice phase obtained with an environmental molecular beam (EMB) apparatus (Kong et al., 2014). The experiment devised by Kong et al. is based on the measurement of $\mathrm{D}_{2} \mathrm{O}$ molecular intensities originating from the surface of a $\mathrm{H}_{2} \mathrm{O}$-ice slab subject to previous $\mathrm{D}_{2} \mathrm{O}$ deposition. Here we evaluate the observed $D_{2} \mathrm{O}$ desorption recorded by the EMB apparatus to investigate the sensitivity of the observed molecular fluxes to potential non-accommodation events. To do this, we use two molecular flux modelling schemes that approximate the evaporation from a disordered interface assumed to consist of an ideal mixture of $\mathrm{D}_{2} \mathrm{O}$ and $\mathrm{H}_{2} \mathrm{O}$ : one accounting for evaporative losses only, and one that additionally accounts for bulk accommodation events.

The molecular flux models are predicated upon proportionality between the detected $\mathrm{D}_{2} \mathrm{O}$ flux from the sample surface and the surface layer $\mathrm{D}_{2} \mathrm{O}$ concentration $I \propto N_{\mathrm{D}_{2} \mathrm{O}}$. In the experimental set-up, $\mathrm{D}_{2} \mathrm{O}$ is first deposited onto an initially $\mathrm{D}_{2} \mathrm{O}$-free sample by the molecular beam at rate $\Gamma$ for a short time interval $\left[t_{1}, t_{2}\right]$. After this, the number concentration $N_{\mathrm{D}_{2} \mathrm{O}}$ in the surface layer decreases as the molecules simultaneously evaporate from the surface or accommodate into the bulk ice. The change rate of $N_{\mathrm{D}_{2} \mathrm{O}}$ can thus be written as

$$
\frac{\mathrm{d} N_{\mathrm{D}_{2} \mathrm{O}}}{\mathrm{d} t}=\left\{\begin{array}{cl}
0 ; & t<t_{1} \\
\Gamma-k_{\mathrm{sum}} N_{\mathrm{D}_{2} \mathrm{O}} ; & t_{1}<t<t_{2} \\
k_{\mathrm{sum}} N_{\mathrm{D}_{2} \mathrm{O}} ; & t_{2}<t
\end{array}\right.
$$

where the $\mathrm{D}_{2} \mathrm{O}$ deposition takes place for times $t_{1}<t<t_{2}$, and $k_{\text {sum }}$ is the sum of the evaporation and accommodation rate constants $k_{\text {sum }}=k_{\mathrm{e}}+k_{\alpha}$. Integration of Eq. (2.2) yields the time-dependent surface concentration

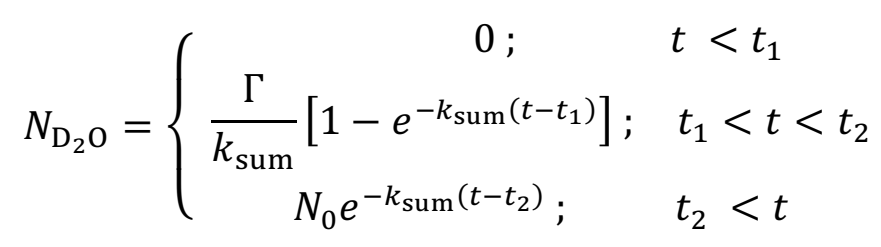

where $N_{0}=\frac{\Gamma}{k_{\text {sum }}}\left[1-e^{-k_{\text {sum }}\left(t_{2}-t_{1}\right)}\right]$ is the surface concentration at $t=t_{2}$ when the beam is switched off. The detected intensity is connected to $N_{\mathrm{D}_{2} \mathrm{O}}$ through the evaporation flux as

$$
I(t)=A \times B \times k_{\mathrm{e}} N_{\mathrm{D}_{2} \mathrm{O}}(t),
$$

where the pre-factor $B$ is determined from the known experimental settings and includes e.g. the slab-to-detector transmission efficiency (see Supplementary Information, $\mathrm{SI}$ ), $k_{\mathrm{e}}$ is the 
evaporation rate constant, and $A$ is a fitting parameter. Here we use the standard Knudsen evaporation rate for $k_{\mathrm{e}}$ as

$$
k_{e}=X \frac{\langle v\rangle C_{S}}{4},
$$

where $\langle v\rangle$ is the mean thermal velocity of gas-phase $\mathrm{D}_{2} \mathrm{O}$ molecules, $C_{s}$ is the saturation vapour concentration over the surface, and $X$ is a parameter accounting for unit conversions and mixing with $\mathrm{H}_{2} \mathrm{O}$ molecules (see $\mathrm{SI}$ ). This approach is chosen to prevent introducing additional experimental uncertainties from Arrhenius-type evaporation rates derived from fits to the measurement data (see Fig. 5b in Kong et al. 2014). Unlike in the previous studies, we do not need to fit the thermal evaporation rate, but can predict it "bottom-up" if the saturation vapour pressure of $\mathrm{D}_{2} \mathrm{O}$ and the thickness of the disordered layer as a function of temperature can be estimated. After removing the average background signal, $I_{n}(t)$ is fit to data recorded at 12 temperatures spanning $170-200 \mathrm{~K}$ using two approaches. First, we apply an evaporation-only model in which $k_{\text {sum }}=k_{\mathrm{e}}$ and a single parameter $A$ is fit; second, an evaporation and bulk accommodation model is used, in which $k_{\text {sum }}=k_{\mathrm{e}}+k_{\alpha}$ and both $A$ and the bulk accommodation rate $k_{\alpha}$ are fit. These fits are used to assess how well the data can be described by such models, and how reliably the accommodation rate constant $k_{\alpha}$ can be constrained from such data.

\section{Results and discussion}

\subsection{Molecular definition of surface and bulk accommodation from MD simulations}

A condensing gas phase molecule can be considered "accommodated" when its physical state is not distinguishable from that of surface (in case of $\alpha_{s}$ ) or bulk (in case of $\alpha_{b}$ ) phase molecules. This axiom is here used to develop criteria for the accommodated states in the disordered surface phase and in the bulk ice. First, we examine the ice slab (excluding the condensing molecule) to characterize the tetrahedrality parameter $q$ and the interaction energy $E$ (Section 2.1) of molecules present in the surface layer and in the bulk ice. We use these data to define the "accommodated" (AC) state, and to determine the fraction of condensing molecules that enter the so-defined AC sub-spaces. 


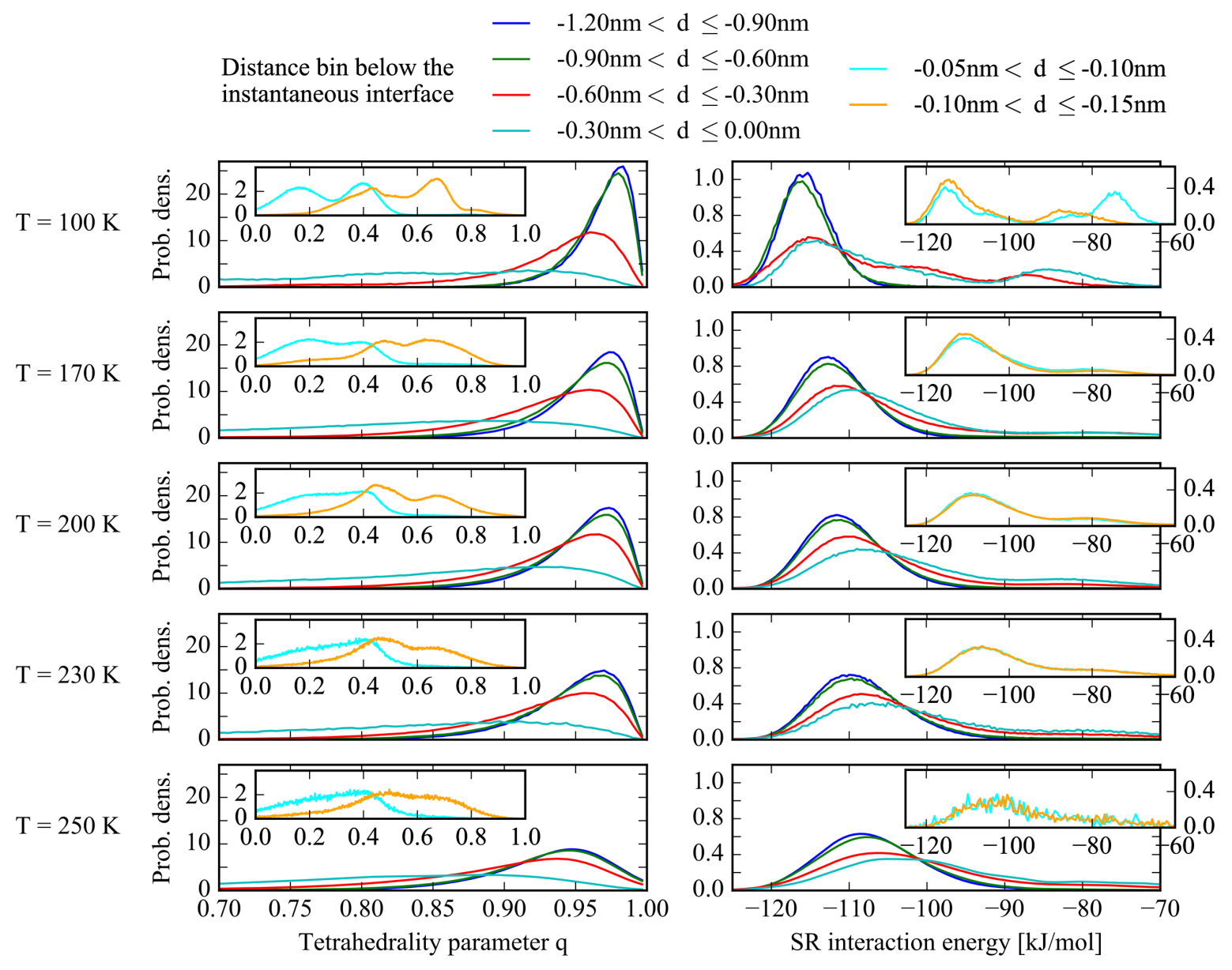

Figure 2: Tetrahedrality parameter and interaction energy distributions for $3 \AA$ thick layers (distance bins) below the instantaneous interface for all temperatures simulated. The insets show tetrahedrality parameter and interaction energy distributions for two thinner layers of $0.5 \AA$ thickness right below the instantaneous interface.

Figure 2 shows the distributions of the tetrahedrality parameter and the interaction energy of the slab molecules at different distances from the instantaneous interface. As expected, the distributions display slightly increasing maxima upon decreasing temperature and small shifts towards higher tetrahedrality parameters and more negative interaction energies. At the same time, they overlap significantly for all layers and all temperatures, except for a narrow binning of $0.5 \AA$ in the top-most layers (insets in Fig. 2), where the order parameter reflects the disorder close to the interface. These results are consistent with those obtained by Gladich et al. (Gladich et al., 2011) who used the same order parameter to characterize the surface layer and investigated the anisotropic surface self-diffusion within the quasi-liquid layer at different temperatures using a six-site model of water. 
Figure 3 shows the medians of the $q$ and $E$ distributions (Fig. 2) at the different temperatures together with the spread indicating the $25^{\text {th }}$ and $75^{\text {th }}$ quartiles for the three top-most layers of $3 \AA$ thickness. The distribution of tetrahedrality parameters in the top-most layer differ slightly from those of the other layers, while the interaction energy distributions overlap for all layers. This observation is valid almost independent of temperature. These results indicate that key parameter differentiating the surface- and bulk-accommodated states is the tetrahedrality parameter, while in terms of the interaction energy these two states are almost indistinguishable from each other.
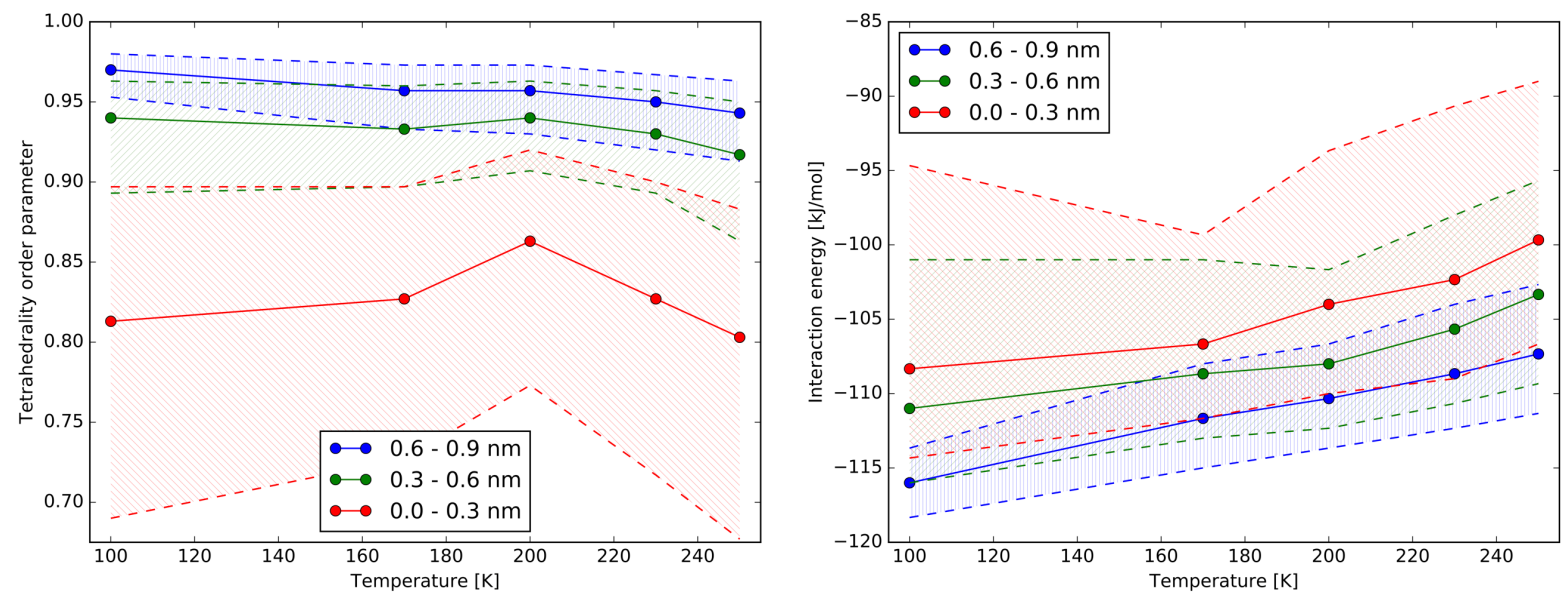

Figure 3: Medians of the distributions of tetrahedrality parameters (left) and the interaction energies (right) as functions of temperature for the three top-most $3 \AA$-layers. The hatched areas depict the width of the distributions as 25-75 quartiles.

Based on the results above, it is clear that the criteria for both surface and bulk accommodation must be temperature-dependent, but also that defining the accommodated states is not unambiguous even for the slab molecules. We define AC criteria for both the surface and the bulk accommodated states, given by the medians of the distributions of $q$ and $E$ of the top-most and the $3^{\text {rd }}$ layer ( $0.6-0.9 \mathrm{~nm}$ below the interface), respectively as presented in Fig. 3. The specific values obtained are listed in Table 1 for all temperatures. These values can be discussed in light of the desorption energy $E_{\text {des }}=42 \pm 8 \mathrm{~kJ} \mathrm{~mol}^{-1}$ and the energy related to the incorporation of water molecules into the ice structure $E_{1}=6 \pm 5 \mathrm{~kJ} \mathrm{~mol}^{-1}$ (interpreted here as the energy associated with the bulk accommodation) reported by Kong et al. (2014) based on adsorption-model fits to the same data as will be discussed in Sect. 3.3. Specifically, it is interesting that the difference in $E$ between the surface and the bulk accommodated 
states is $5-8 \mathrm{~kJ} \mathrm{~mol}^{-1}$ and thus comparable in magnitude to the $E_{1}$ value by Kong et al. (2014), while being significantly smaller than the desorption energy.

\begin{tabular}{|c|c|c|c|c|}
\hline \multirow[b]{2}{*}{$T[\mathrm{~K}]$} & \multicolumn{2}{|c|}{$\begin{array}{l}\text { Surface }(-3 \AA<d<=0 \AA \text { ) } \\
\text { AC sub-space (medians) }\end{array}$} & \multicolumn{2}{|c|}{$\begin{array}{c}\text { Bulk }(-9 \AA<d<=6 \AA \AA) \\
\text { AC sub-space (medians) }\end{array}$} \\
\hline & $q$ & $E[\mathrm{~kJ} / \mathrm{mol}]$ & $q$ & $E[\mathrm{~kJ} / \mathrm{mol}]$ \\
\hline 100 & 0.813 & -108.3 & 0.970 & -116.0 \\
\hline 170 & 0.827 & -106.7 & 0.957 & -111.7 \\
\hline 200 & 0.863 & -104.0 & 0.957 & -110.3 \\
\hline 230 & 0.827 & -102.3 & 0.950 & -108.7 \\
\hline 250 & 0.803 & -99.7 & 0.943 & -107.3 \\
\hline
\end{tabular}

Table 1: Definition of "surface-accommodated" and "bulk-accommodated" sub-spaces using the medians of $q$ - and $E$-distributions. The sub-space definitions is based on a subdivision into $3 \AA$ layers (about one molecular diameter) parallel to the instantaneous interface.

It is important to note that while the statistics over a large number of molecules give rise to the average properties of the slab layers, the individual molecules are subject to pronounced thermal fluctuations - the magnitude of which increases with temperature. A bulk molecule can be in an $A C$ state with fluctuations into non-AC states and vice versa. The exchange frequencies between the $\mathrm{AC}$ (see Table 1) and non-AC states lie in the range of $0 \leq f_{e x} \leq$ $30 \mathrm{~ns}^{-1}$ for all temperatures, with a tendency of higher frequencies in the centre of the slab and lower frequencies in the surface layer (see SI). We interpret this finding as a result of stronger bonds at crystal sites than at the surface, in analogy to the frequency of a harmonic oscillator depending on the force constant. This picture also explains the trend in the AC state definition with temperature since the mean square displacement of molecules about crystal sites due to thermal motion is directly proportional to temperature, assuming a harmonic potential. Enhanced spatial fluctuations would in turn cause fluctuations in interaction energy and tetrahedrality parameter that cause fluctuations into and out of a given AC sub-space. Closer to the interface and at higher temperatures, the approximation of harmonic potentials naturally cannot be expected to hold while the qualitative considerations are unaffected.

\subsection{The kinetics of condensing molecules and timescales related to accommodation}

Let us now discuss the kinetics and time scales of the accommodation process of a condensing vapour phase molecule implementing the above criteria, as this will be critical for linking the 
theoretical findings to experimental data. Furthermore, the time scales associated with the accommodation phenomena are expected to be highly dependent on temperature, and hence important when discussing the potential explanations to the observed temperature trends.
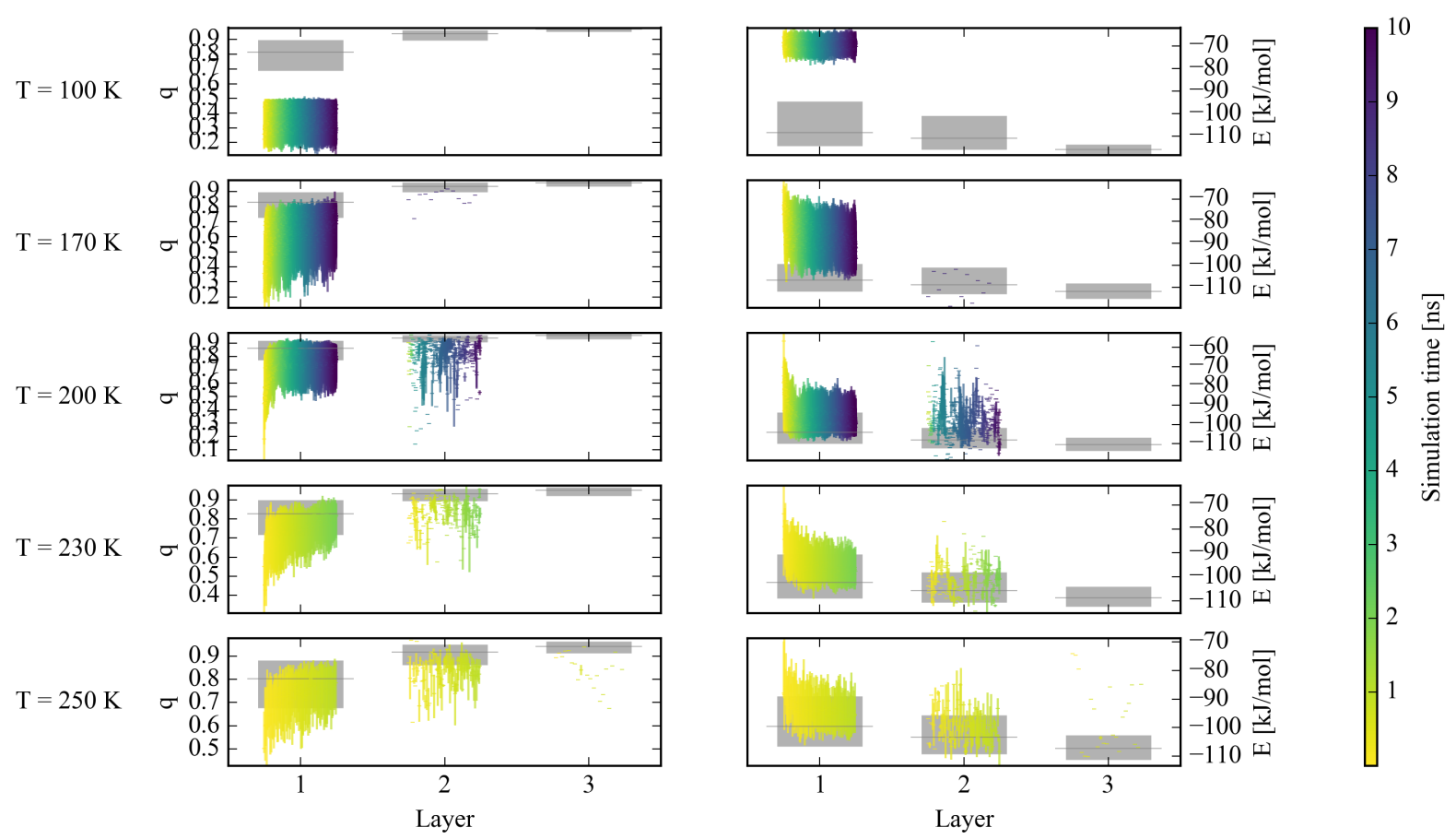

Figure 4: Characteristic values of interaction energy and tetrahedrality parameter distributions of the slab (gray bars) and condensed molecules (coloured lines). The bars indicate the $25^{\text {th }}$ to $75^{\text {th }}$ percentiles, the median is indicated with a thin line; condensing molecules' distributions are obtained as median and $25^{\text {th }}$ to $75^{\text {th }}$ percentiles of up to 100 molecules at times encoded in their colour. Absence of condensing molecules' distribution lines at low temperatures and in the lower layers indicates that no condensing molecules have diffused to the layer in question within the respective simulation time.

Figure 4 shows a comparison of the $q$ and $E$ distributions of the condensing molecules throughout the simulation time as compared with those of the slab molecules (see Fig. 2) as a function of temperature. The distributions for slab molecules (gray) are statistics over the whole trajectory, represented by mean and quartiles, while the distributions of the condensed molecules represent statistics over up to 100 simulations for each time bin of 10 ps. Every coloured line thus represents the distributions of tetrahedrality parameter and interaction energy, respectively, with its colour encoding the time bin along the trajectory. It can be seen that while the distributions overlap for temperatures $T>100 \mathrm{~K}$, the condensed molecules have generally lower tetrahedrality parameters and less negative interaction energies than the slab molecules in the same layer, with the distributions of the condensed molecules shifting 
towards those of the bulk molecules over time. Furthermore, it is notable that the condensing molecules do not diffuse beyond the first layer for $T=100 \mathrm{~K}$ within our simulation time of 10 ns while they distribute over first, second, and third layer for $T=250 \mathrm{~K}$ even within shorter simulation times of $1 \mathrm{~ns}$. These results suggest that the time scales of both surface and bulk accommodation are indeed highly dependent on temperature. Once the condensing molecule adsorbs onto the disordered surface layer, it will thermally equilibrate with its nearest neighbours within some 10 picoseconds (Neshyba et al., 2009a), and subsequently its interaction energy and tetrahedrality parameter will gradually become indistinguishable from the slab molecules. Defined this way, the surface accommodation process is estimated to happen within time scales of roughly $1 \mathrm{~ns}(T=250,230 \mathrm{~K}), 10 \mathrm{~ns}(T=200 \mathrm{~K}), 45 \mathrm{~ns}(T=170 \mathrm{~K})$, and $250 \mathrm{~ns}(\mathrm{~T}=100 \mathrm{~K})$, based on the decay of the time correlation functions of $q$ and $E$ (see Fig. S2 and Tab. S5 in the SI).

Uptake into the disordered interface thus plays a decisive role being the first step in the accommodation process: the subsequent bulk accommodation of the initially-labelled molecule into the bulk crystal becomes equivalent to the concept of equilibrium exchange processes of slab molecules between the bulk crystal and its disordered surface layer. Based on literature values of ice growth rates in Xu et al. (2016), the time scale of the bulk accommodation from the surface layer is hence expected to vary considerably, ranging from nanoseconds to seconds between 126 and $262 \mathrm{~K}$, showing a minimum around $250 \mathrm{~K}$ (Xu et al., 2016). These estimates are also in line with our simulations, where some molecules reached the accommodated state within $10 \mathrm{~ns}$ while others did not, and the median properties of the condensing molecules were closest to the slab values at $250 \mathrm{~K}$ (see Fig. 4). Specifically, the condensing molecules are observed not to diffuse beyond the top-most layer for all temperatures, but rather stay at the interface for lower temperatures beyond time scales covered by the simulations presented here. Simulation studies of equilibration kinetics would require ergodic sampling and thus much longer simulation times of the order of microseconds. Furthermore, these expected accommodation time scales are all considerably shorter than the ms time scales probed by the EMB experiment discussed in detail below. An schematic overview of the different time scales is presented in Fig. 5. 


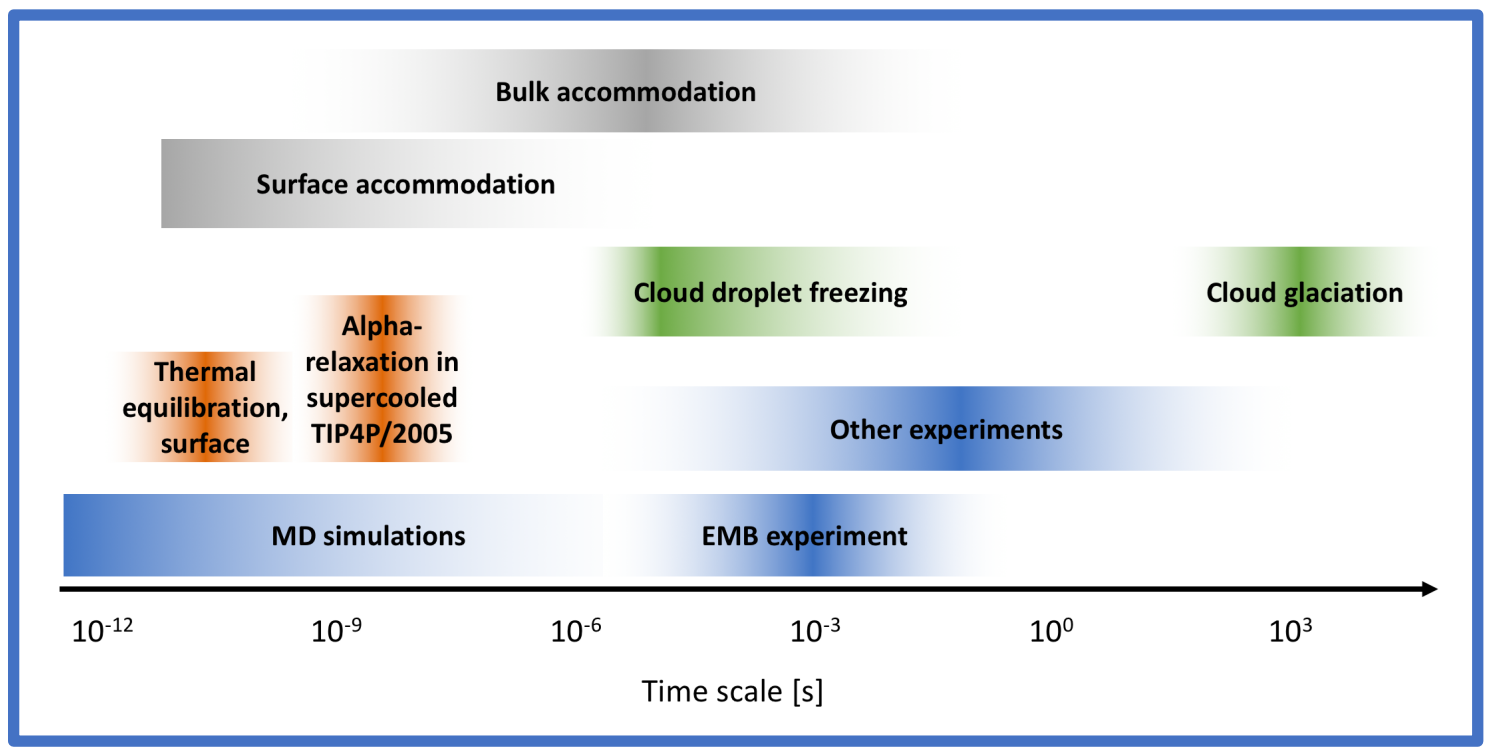

Figure 5: A schematic illustrating the time scales of different methods and phenomena of interest in the context of accommodation processes: typical time scales of MD simulations and EMB experiments by Kong et al. (2014) considered here, estimates for time scales of surface and bulk accommodation processes, surface relaxation time scales (Neshyba et al., $2009 \mathrm{~b}$ ), structural relaxation time scale in simulations of supercooled bulk water (Wikfeldt et al., 2011), and typical time scales of associated cloud-microphysical processes (Korolev et al., 2017).

To illustrate the accommodation process in more detail, Fig. 1 shows typical example snapshots from a simulation trajectory at $200 \mathrm{~K}$ visualising a gas-phase molecule condensing onto the ice surface (Fig. 1a), and the interaction energy and the tetrahedrality parameter of the molecule as a function of time (Fig. 1b). The instantaneous interface is indicated in blue and the condensing molecule is followed while penetrating this interface. Initial adsorption onto the surface layer, indicated by a drop in interaction energy to around $-50 \mathrm{~kJ} / \mathrm{mol}$, takes place within the first few ps of the simulation. This is followed by reorientation movements of the condensing molecule accociated with fluctuations in interaction energy and tetrahedrality parameter. At about 182 ps, there is a transition into a lower energy, i.e. a stronger binding, state at an energy fluctuating about $-90--100 \mathrm{~kJ} / \mathrm{mol}$. We attribute the different interaction energy states to different hydrogen-bonded ( $\mathrm{H}$-bonded) configurations. The snapshot at $t=$ 182 ps shows the condensing molecule having penetrated the instantaneous surface, accompanied by an exchange process with a molecule from the surface layer, $\mathrm{H}$-bonding to the condensed molecule. The resulting near-tetrahedral coordination of the condensed molecule gives rise to at temporarily very high tetrahedrality parameter and a very negative interaction energy, as seen in Fig. $1 \mathrm{~b}$. 
To further examine the potential bulk accommodation in our simulations, results from a first passage time (FPT) analysis are shown in Fig. 6, where the cumulative fraction of simulations exhibiting a surface (Fig. 6a) or bulk (Fig. 6b) accommodation event (following the criteria in Table 1) for the first time is presented as a function of time for each temperature. While the cumulative FPT distributions for all temperatures change towards a lower fraction of molecules being accommodated in the bulk as compared with the surface, this change is observed to be most pronounced for $\mathrm{T}=170 \mathrm{~K}$. Simulations at $\mathrm{T}=100 \mathrm{~K}$ were not run long enough to resolve more accommodation events. We emphasize that the FPT accommodation rate, corresponding to the slope of the FPT distributions, seems to undergo a rather dramatic change between $\mathrm{T}=170 \mathrm{~K}$ and $200 \mathrm{~K}$ when the criteria are changed from weaker to stronger. These results further emphasize the results on the strong temperature-dependence of the accommodation processes (see also Table S1 and Fig. 4): while the time scales of surface and bulk accommodation are similar for temperatures between 200 and $250 \mathrm{~K}$, they diverge at lower temperatures where the bulk processes become drastically slower. Both time scales generally shorten with increasing temperature in the temperature range probed here (100 $250 \mathrm{~K})$. 


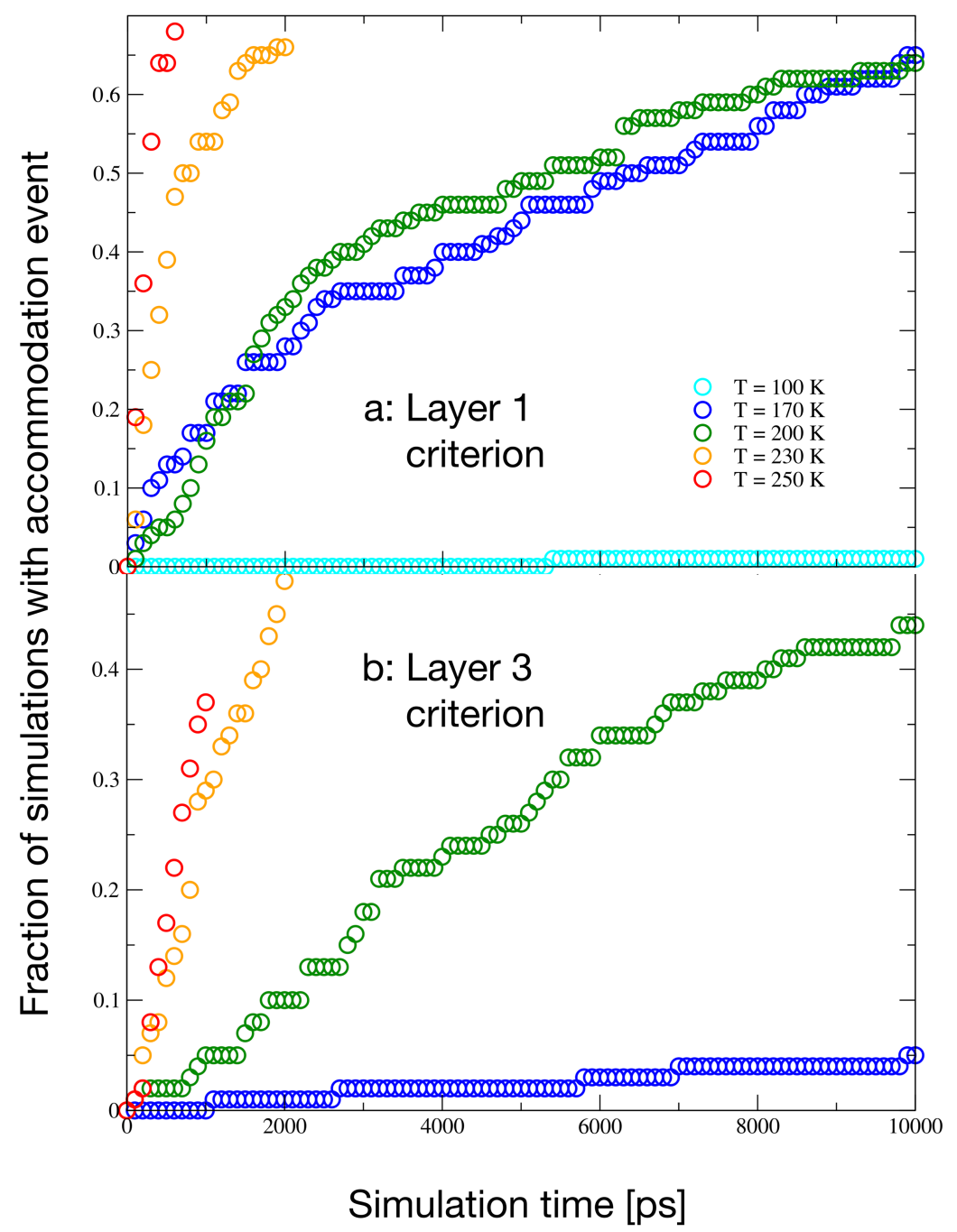

Figure 6: First passage time (FPT) analysis. Fraction of 100 simulations with a condensing vapour phase molecule that exhibit an accommodation event as a function of time (100 ps time bins). An accommodation event is identified when the condensing molecule enters the accommodated space defined for the respective temperature for the first time. (a) AC criteria found from the medians of interaction energy and tetrahedrality parameter in layer 1 $(-0.3 \mathrm{~nm}<d \leq 0.0 \mathrm{~nm})$; (b) corresponding criteria found for layer $3(-0.6 \mathrm{~nm} \leq d<$ $-0.9 \mathrm{~nm})$, see Table 1 .

The frequency of exchange between accommodated and non-accommodated states discussed above can further be compared to the FPT accommodation rate. While the exchange frequencies are found in the range of $0 \leq f_{e x} \leq 30 \mathrm{~ns}^{-1}$, the FPT accommodation rates cover a wider interval of roughly $0 \leq f_{F P T} \leq 130 \mathrm{~ns}^{-1}$, with good agreement at low temperatures where the frequencies are of the orders $0.1-10 \mathrm{~ns}^{-1}$ (see also $\mathrm{SI}$ ).

Along the trajectories of the condensing molecules, the tetrahedrality parameter $q$ is roughly anti-correlated with the interaction energy $E$ in the condensed phase (see Fig. 7 for $200 \mathrm{~K})$. Generally, $q$ increases with more negative distances together with increasingly 
negative $E$, indicating the structural order to increase the further the molecule penetrates the surface layer towards the bulk. As a summary, tetrahedrality parameter, interaction energy, and the distance to the instantaneous interface are not strictly independent: Generally, molecules with the strongest binding energy tend to correlate with the highest values of $q$ and lye deepest down in the surface layer. The choice of these parameters for defining the accommodated states within the surface and the bulk are, however, justified e.g. by the distinctly different behaviour of the bulk vs. surface visible in the tetrahedrality parameter but not in the interaction energy (see Fig. 3).

\subsection{Interpretation of the EMB data by evaporation-accommodation modelling}

Our sensitivity analysis utilizing one- and two-parameter fits (see Methods section and SI), respectively, clearly shows that evaporation alone cannot explain the depletion of $D_{2} \mathrm{O}$ at the ice surface, particularly for temperatures below $190 \mathrm{~K}$, in agreement with earlier results by Kong et al 2014. The evaporation rate is, on the other hand, a major source of uncertainty (see $\mathrm{SI})$, due to uncertainties in the vapour pressure parameterizations. We find that an evaporation rate calculated from Knudsen theory with the vapour pressure of $\mathrm{D}_{2} \mathrm{O}$ ice as obtained by Matsuo et al (Matsuo et al., 1964) can fit the data to same accuracy as the Arrhenius-type evaporation rate employed by Kong et al whose fit yields a desorption activation energy of (42 \pm 9) kJ/mol (Kong et al., 2014) (cf. SI).

Reproducing the results obtained earlier, we investigated the theoretical maximum fraction of molecules that can evaporate from the surface after deposition within a certain time interval, without any accommodation taken into account. Fig. 7 shows the evaporated

fraction $F_{e}\left(T, t_{\text {end }}\right)$ as a function of temperature $T$ and elapsed time after deposition $t_{\text {end }}$. This analysis suggests that an experimental time window of $60 \mathrm{~ms}$ might be insufficient and could potentially lead to an artificially high value of $\alpha$ at low temperatures, a concern raised earlier by Kong et al., 2014. This suggests that the experimental data is possibly insufficient to conclusively constrain the bulk accommodation process. Furthermore, as both evaporation and accommodation processes are temperature-dependent, it is challenging to disentangle their effects on the observed signal. For instance, the fact that more $\mathrm{D}_{2} \mathrm{O}$ desorbed from the surface is detected at higher temperatures may be due to increased, highly uncertain, 
evaporation rate, instead of reflecting reduced bulk accommodation. These results suggest that techniques that are able to directly characterize the surface states would be favourable compared to indirect assessments of surface processes. Further development of the EMB technique towards longer measurement time intervals and improved signal-to-noise ratio, on the other hand, is a promising alternative as this allows the direct observation of the sorption kinetics.

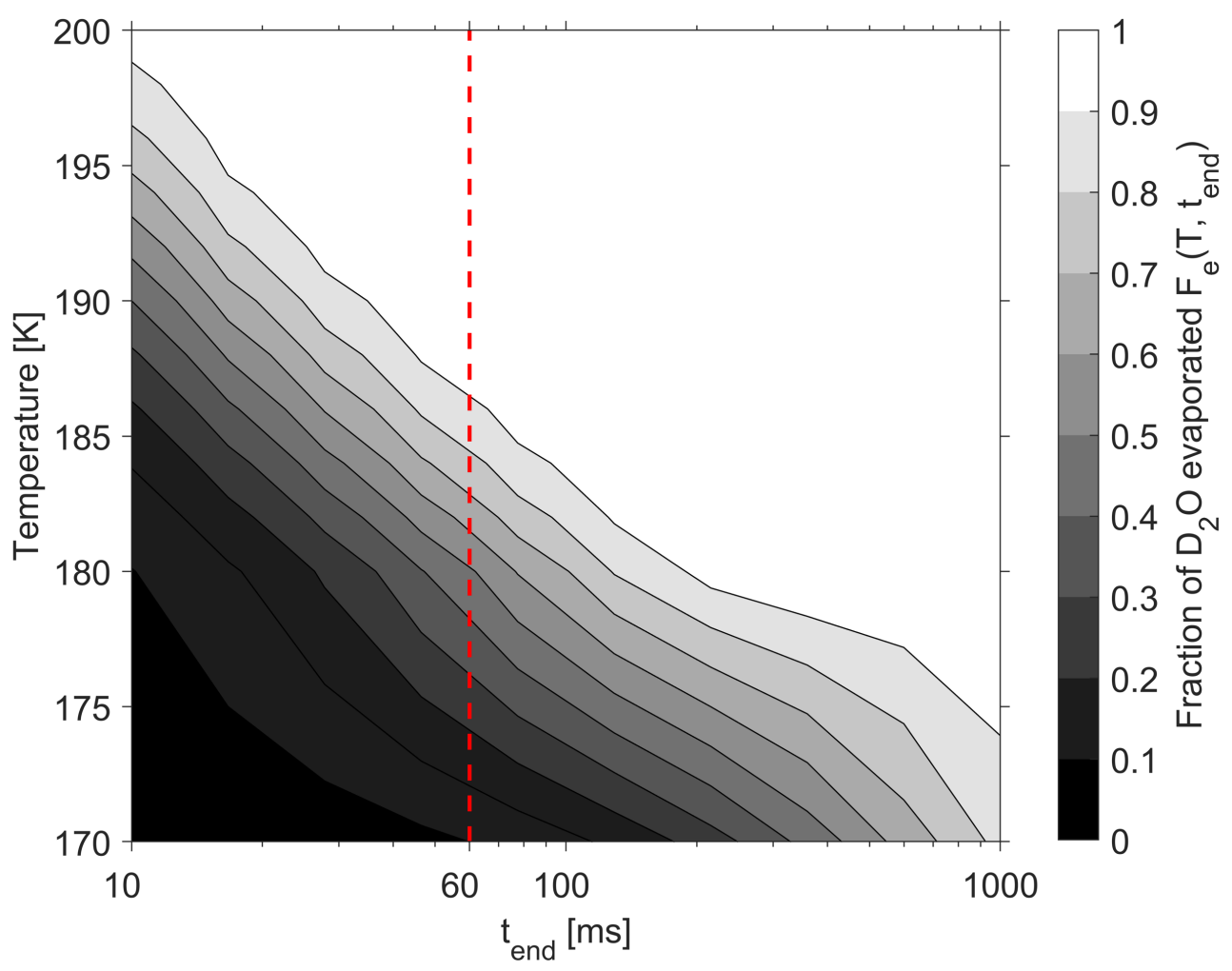

Figure 7: Contour plot of the theoretical fraction of initially deposited $\mathrm{D}_{2} \mathrm{O}$ molecules $F_{e}\left(T, t_{\text {end }}\right)$ that has evaporated at temperature $T$ after time $t_{\text {end, }}$ disregarding accommodation. The dashed red line marks the observation time window in the EMB experiment by Kong et al.

\section{Conclusions}

In the present study we set out to characterise the accommodation process using molecular dynamics simulations. The simulations suggest that the definition of the accommodated state plays a decisive role in characterizing and interpreting the accommodation of water vapour on ice due to the presence of the disordered layer on top of ice surfaces. Molecules within this layer undergo fluctuations between more ordered, ice-like states and less ordered surface states. Therefore, even if a condensing vapour-phase molecule is not built into the bulk ice lattice immediately after impinging the surface, it can be considered accommodated to the 
surface as soon as its energetics and orientational order cannot be distinguished from those of the surrounding surface molecules at equilibrium. Recognizing the surface layer of ice to be disordered to an extend depending on the temperature difference to the melting temperature, we investigate distributions of the molecular tetrahedrality parameter and the interaction energy as functions of depth below the instantaneous interface and as functions of temperature. Based on these distributions, we define the space of accommodated states. We emphasize that even under equilibrium conditions, molecules that are part of the simulated ice slab fluctuate into and out of these so defined accommodated states.

The interaction energy is naturally dominated by the hydrogen bond state of the molecule considered. From our analysis of energy distributions, the differences associated with the transition between surface layer and bulk crystal widely overlap, and the associated energy change is thus very difficult to constrain from measurements where also evaporation (with an uncertain evaporation rate) takes place. While it is evident that the experimental data by Kong et al. cannot be described by evaporation alone, bulk accommodation is difficult to reliably be dissected from the data as the signal is heavily influenced by the uncertainty in the evaporation rate.

We find here that the distribution of molecular interaction energies does not provide a sensitive measure in the context of accommodation while the tetrahedrality parameter is a much more suitable means to distinguish surface and bulk states. This result indicates that experiments probing bulk accommodation from the surface layer should preferably be able to resolve structural changes on molecular scales.

Having characterized the slab molecules, we follow the evolution of individual molecules condensing onto the surface while their properties relax towards those of the slab molecules. The above analysis demonstrates a strong temperature dependence of the surface and bulk accommodation kinetics: The time correlation functions yield relaxation times for surface layer accommodation of the order of $1 \mathrm{~ns}$ at $250 \mathrm{~K}$ and $250 \mathrm{~ns}$ at $100 \mathrm{~K}$, while literature data on ice crystal growth rates suggests bulk accommodation timescales ranging from ns to $\mathrm{s}$ in a similar temperature range (Xu et al., 2016). This highlights the need for being able to adjust the time window probed in any experiments targeted to observe the kinetics of water vapour accommodation as a function of temperature.

Our first passage time analysis shows a rather pronounced drop in the accommodation rate below $\mathrm{T}=200 \mathrm{~K}$ and on the time scales resolved in this study, when 
results obtained with the stronger criteria, deduced from bulk molecular states, are compared to those obtained with the weaker criteria that was deduced from molecular states in the surface layer. Thus, we argue that depositional ice growth can be thought of as a two-step process, with an initial adsorption to the disordered surface layer and subsequent accommodation into the bulk crystal on longer time scales.

We note that the concept of accommodation of vapour phase molecules into an ice crystal is tightly interconnected to crystal growth rather than to equilibrium dynamics. Measurements of ice crystal growth show that the growth rate at atmospherically relevant conditions is orders of magnitudes faster than any net condensation flux for a wide temperature range $(100-280 \mathrm{~K})$ (Seinfeld and Pandis, 2006). This means that a possible energy barrier to accommodation to the ice lattice (if any) is so small that it is not expected to play any role in the atmospheric context. This makes the uptake of condensing molecules onto the surface the rate-limiting process. In this case the relevant parameter characterizing accommodation is the sticking coefficient of vapour molecules colliding with the surface, for which numerous simulation studies have indicated a value of unity for the liquid water surface (Julin et al., 2013; Schlesinger et al., 2016; Varilly and Chandler, 2013). At temperatures close to the melting temperature, we speculate that this value shouldn't be different for the premelting layer of ice than for the surface of the liquid and thus expect a value of unity for the surface accommodation also in this case.

\section{Supporting Information Description}

The Supporting Information (SI) contains an evaluation of the flux models for the reanalysis of EMB data, details on the interaction energy and tetrahedrality parameter distributions from MD simulations, interaction energy and tetrahedrality parameter time correlation functions of condensing molecules, and an analysis of exchange frequencies between accommodated states.

\section{Acknowledgements}

We gratefully acknowledge funding through the K. \& A. Wallenberg foundation, project AtmoRemove (2015.0162). The simulations were performed on resources provided by the Swedish National Infrastructure for Computing (SNIC) at the National Supercomputer Centre 
(NSC) and the High Performance Computing Centre North (HPC2N). D.S. wants to thank Evgeni Zapadinski for useful comments on an early version of the manuscript. T.O. thanks the ÅForsk foundation (project 18-334) for financial support.

\section{References}

Abascal, J., Sanz, E., García Fernández, R. and Vega, C.: A potential model for the study of ices and amorphous water: TIP4P/Ice, J. Chem. Phys., 122(23), 234511, 2005.

Abraham, M. J., Murtola, T., Schulz, R., Páll, S., Smith, J. C., Hess, B. and Lindahl, E.: GROMACS: High performance molecular simulations through multi-level parallelism from laptops to supercomputers, SoftwareX, 1-2, 19-25, doi:https://doi.org/10.1016/j.softx.2015.06.001, 2015.

Bartels-Rausch, T., Jacobi, H.-W., Kahan, T. F., Thomas, J. L., Thomson, E. S., Abbatt, J. P., Ammann, M., Blackford, J. R., Bluhm, H., Boxe, C. and others: A review of air-ice chemical and physical interactions (AICl): liquids, quasi-liquids, and solids in snow, Atmospheric Chem. Phys., 14(3), 1587-1633, 2014.

Brown, D. E., George, S. M., Huang, C., Wong, E. K. L., Rider, K. B., Smith, R. S. and Kay, B. D.: $\mathrm{H} 2 \mathrm{O}$ Condensation Coefficient and Refractive Index for Vapor-Deposited Ice from Molecular Beam and Optical Interference Measurements, J. Phys. Chem., 100(12), 4988-4995, doi:10.1021/jp952547j, 1996.

Bryson, C. E., Cazcarra, V. and Levenson, L. L.: Condensation coefficient measurements of H2O, N2O, and CO2, J. Vac. Sci. Technol., 11(1), 411-416, doi:10.1116/1.1318639, 1974.

Bussi, G., Donadio, D. and Parrinello, M.: Canonical sampling through velocity rescaling, J. Chem. Phys., 126(1), 014101, doi:10.1063/1.2408420, 2007.

Chaix, L., Allanic, A. and Rossi, M. J.: Heterogeneous Chemistry of $\mathrm{HOBr}$ on Different Types of Ice and on Ice Doped with $\mathrm{HCl}, \mathrm{HBr}$, and $\mathrm{HNO} 3$ at $175 \mathrm{~K}<\mathrm{T}<215 \mathrm{~K}$, J. Phys. Chem. A, 104(31), 7268-7277, doi:10.1021/jp001018z, 2000.

Choularton, T. W. and Latham, J.: Measurements of the deposition coefficient for ice, and its application to cirrus seeding, Q. J. R. Meteorol. Soc., 103(436), 307-318, doi:10.1002/qj.49710343608, 1977.

Conde, M., Vega, C. and Patrykiejew, A.: The thickness of a liquid layer on the free surface of ice as obtained from computer simulation, J. Chem. Phys., 129(1), 014702, 2008.

Dash, J., Rempel, A. and Wettlaufer, J.: The physics of premelted ice and its geophysical consequences, Rev. Mod. Phys., 78(3), 695, 2006. 
Delval, C. and Rossi, M. J.: Influence of Monolayer Amounts of HNO3 on the Evaporation Rate of H2O over Ice in the Range 179 to $208 \mathrm{~K}$ : A Quartz Crystal Microbalance Study, J. Phys. Chem. A, 109(32), 7151-7165, doi:10.1021/jp0505072, 2005.

Delval, C., Fluckiger, B. and Rossi, M. J.: The rate of water vapor evaporation from ice substrates in the presence of $\mathrm{HCl}$ and $\mathrm{HBr}$ : implications for the lifetime of atmospheric ice particles, Atmospheric Chem. Phys., 3(4), 1131-1145, doi:https://doi.org/10.5194/acp-31131-2003, 2003.

Errington, J. R. and Debenedetti, P. G.: Relationship between structural order and the anomalies of liquid water, Nature, 409(6818), 318, 2001.

Fluckiger, B. and Rossi, M. J.: Common Precursor Mechanism for the Heterogeneous Reaction of $\mathrm{D} 2 \mathrm{O}, \mathrm{HCl}, \mathrm{HBr}$, and $\mathrm{HOBr}$ with Water Ice in the Range 170-230 K: Mass Accommodation Coefficients on Ice, J. Phys. Chem. A, 107(20), 4103-4115, doi:10.1021/jp021956u, 2003.

Frenkel, D. and Smit, B.: Understanding Molecular Simulation: From Algorithms to Applications, Elsevier., 2001.

Fuchs, N. A. and Sutugin, A. G.: Highly Dispersed Aerosols, Ann Arbor Science., 1970.

Gladich, I., Pfalzgraff, W., Maršálek, O., Jungwirth, P., Roeselová, M. and Neshyba, S.: Arrhenius analysis of anisotropic surface self-diffusion on the prismatic facet of ice, Phys. Chem. Chem. Phys., 13(44), 19960-19969, doi:10.1039/C1CP22238D, 2011.

Haynes, D. R., Tro, N. J. and George, S. M.: Condensation and evaporation of water on ice surfaces, J. Phys. Chem., 96(21), 8502-8509, doi:10.1021/j100200a055, 1992.

Hienola, J., Kulmala, M. and Laaksonen, A.: Condensation and evaporation of water vapor in mixed aerosols of liquid droplets and ice: numerical comparison of growth rate expressions, J. Aerosol Sci., 32(3), 351-374, doi:10.1016/S0021-8502(00)00085-9, 2001.

Humphrey, W., Dalke, A. and Schulten, K.: VMD: visual molecular dynamics, J. Mol. Graph., 14(1), 33-38, 1996.

Isono, K. and Iwai, K.: Growth Mode of Ice Crystals in Air at Low Pressure, Nature, 223(5211), 1149, doi:10.1038/2231149a0, 1969.

Julin, J., Shiraiwa, M., Miles, R. E., Reid, J. P., Pöschl, U. and Riipinen, I.: Mass accommodation of water: Bridging the gap between molecular dynamics simulations and kinetic condensation models, J. Phys. Chem. A, 117(2), 410-420, 2013.

Kolb, C. E., Cox, R. A., Abbatt, J. P. D., Ammann, M., Davis, E. J., Donaldson, D. J., Garrett, B. C., George, C., Griffiths, P. T., Hanson, D. R., Kulmala, M., McFiggans, G., Pöschl, U., Riipinen, I., Rossi, M. J., Rudich, Y., Wagner, P. E., Winkler, P. M., Worsnop, D. R. and Dowd, C. D. O.: An overview of current issues in the uptake of atmospheric trace gases by aerosols and clouds, Atmospheric Chem. Phys., 10(21), 10561-10605, doi:https://doi.org/10.5194/acp10-10561-2010, 2010. 
Kong, X., Papagiannakopoulos, P., Thomson, E. S., Markovic, N. and Pettersson, J. B.: Water accommodation and desorption kinetics on ice, J. Phys. Chem. A, 118(22), 3973-3979, 2014.

Korolev, A., McFarquhar, G., Field, P. R., Franklin, C., Lawson, P., Wang, Z., Williams, E., Abel, S. J., Axisa, D., Borrmann, S., Crosier, J., Fugal, J., Krämer, M., Lohmann, U., Schlenczek, O., Schnaiter, M. and Wendisch, M.: Mixed-Phase Clouds: Progress and Challenges, Meteorol. Monogr., 58, 5.1-5.50, doi:10.1175/AMSMONOGRAPHS-D-17-0001.1, 2017.

Koros, R. M., Deckers, J. M., Andres, R. P. and Boudart, M.: The sticking probability of water on ice, Chem. Eng. Sci., 21(10), 941-950, doi:10.1016/0009-2509(66)85088-1, 1966.

Kramers, H. and Stemerding, S.: The sublimation of ice in vacuum, Appl. Sci. Res. Sect. A, 3(1), 73-82, doi:10.1007/BF03184673, 1951.

Kulmala, M. and Wagner, P. E.: Mass accommodation and uptake coefficients - a quantitative comparison, J. Aerosol Sci., 32(7), 833-841, doi:10.1016/S0021-8502(00)001166, 2001.

Lamb, D. and Verlinde, J.: Physics and Chemistry of Clouds, Cambridge University Press., 2011.

Leu, M.-T.: Heterogeneous reactions of $\mathrm{N} 2 \mathrm{O} 5$ with $\mathrm{H} 2 \mathrm{O}$ and $\mathrm{HCl}$ on ice surfaces: Implications for Antarctic ozone depletion, Geophys. Res. Lett., 15(8), 851-854, doi:10.1029/GL015i008p00851, 1988.

Limmer, D. T. and Chandler, D.: Premelting, fluctuations, and coarse-graining of water-ice interfaces, J. Chem. Phys., 141(18), 18C505, doi:10.1063/1.4895399, 2014.

Liu, X., Day, D. A., Krechmer, J. E., Brown, W., Peng, Z., Ziemann, P. J. and Jimenez, J. L.: Direct measurements of semi-volatile organic compound dynamics show near-unity mass accommodation coefficients for diverse aerosols, Commun. Chem., 2(1), 98, doi:10.1038/s42004-019-0200-x, 2019.

Lohmann, U., Lüönd, F. and Mahrt, F.: An Introduction to Clouds: From the Microscale to Climate, Cambridge University Press., 2016.

Magee, N., Moyle, A. M. and Lamb, D.: Experimental determination of the deposition coefficient of small cirrus-like ice crystals near $-50^{\circ} \mathrm{C}$, Geophys. Res. Lett., 33(17), doi:10.1029/2006GL026665, 2006.

Matsuo, S., Kuniyoshi, H. and Miyake, Y.: Vapor Pressure of Ice Containing D2O, Science, 145(3639), 1454-1455, doi:10.1126/science.145.3639.1454, 1964.

Miles, R. E. H., Reid, J. P. and Riipinen, I.: Comparison of Approaches for Measuring the Mass Accommodation Coefficient for the Condensation of Water and Sensitivities to Uncertainties in Thermophysical Properties, J. Phys. Chem. A, 116(44), 10810-10825, doi:10.1021/jp3083858, 2012.

Mohandesi, A. and Kusalik, P. G.: Probing ice growth from vapor phase: A molecular dynamics simulation approach, J. Cryst. Growth, 483, 156-163, 2018. 
Neshyba, S., Nugent, E., Roeselová, M. and Jungwirth, P.: Molecular Dynamics Study of IceVapor Interactions via the Quasi-Liquid Layer, J. Phys. Chem. C, 113(11), 4597-4604, 2009a.

Neshyba, S., Nugent, E., Roeselová, M. and Jungwirth, P.: Molecular Dynamics Study of Ice-Vapor Interactions via the Quasi-Liquid Layer, J. Phys. Chem. C, 113(11), 4597-4604, doi:10.1021/jp810589a, 2009b.

Neshyba, S., Adams, J., Reed, K., Rowe, P. M. and Gladich, I.: A quasi-liquid mediated continuum model of faceted ice dynamics, J. Geophys. Res. Atmospheres, 121(23), 2016.

Parrinello, M. and Rahman, A.: Polymorphic transitions in single crystals: A new molecular dynamics method, J. Appl. Phys., 52(12), 7182-7190, doi:10.1063/1.328693, 1981.

Pöschl, U., Rudich, Y. and Ammann, M.: Kinetic model framework for aerosol and cloud surface chemistry and gas-particle interactions \&ndash; Part 1: General equations, parameters, and terminology, Atmospheric Chem. Phys., 7(23), 5989-6023, doi:https://doi.org/10.5194/acp-7-5989-2007, 2007.

Pratte, P., van den Bergh, H. and Rossi, M. J.: The Kinetics of H2O Vapor Condensation and Evaporation on Different Types of Ice in the Range 130-210 K, J. Phys. Chem. A, 110(9), 3042-3058, doi:10.1021/jp053974s, 2006.

Pruppacher, H. R. and Klett, J. D.: Microphysics of Clouds and Precipitation, 2nd ed., Springer Netherlands., 2010.

Schlesinger, D., Sellberg, J. A., Nilsson, A. and Pettersson, L. G.: Evaporative cooling of microscopic water droplets in vacuo: Molecular dynamics simulations and kinetic gas theory, J. Chem. Phys., 144(12), 124502, 2016.

Seinfeld, J. H. and Pandis, S. N.: Atmospheric chemistry and physics: from air pollution to climate change, Second., John Wiley \& Sons., 2006.

Seinfeld, J. H. and Pandis, S. N.: Atmospheric Chemistry and Physics: From Air Pollution to Climate Change, John Wiley \& Sons., 2016.

Sinha, A., Saleh, R., Robinson, E. S., Ahern, A. T., Tkacik, D. S., Presto, A. A., Sullivan, R. C., Robinson, A. L. and Donahue, N. M.: Mass accommodation coefficients of fresh and aged biomass-burning emissions, Aerosol Sci. Technol., 52(3), 300-309, doi:10.1080/02786826.2017.1413488, 2018.

Skrotzki, J., Connolly, P., Schnaiter, M., Saathoff, H., Möhler, O., Wagner, R., Niemand, M., Ebert, $\mathrm{V}$. and Leisner, T.: The accommodation coefficient of water molecules on ice-cirrus cloud studies at the AIDA simulation chamber, Atmospheric Chem. Phys., 13(8), 4451-4466, 2013.

Slater, B. and Michaelides, A.: Surface premelting of water ice, Nat. Rev. Chem., 3(3), 172188, doi:10.1038/s41570-019-0080-8, 2019. 
Tolbert, M. A. and Middlebrook, A. M.: Fourier transform infrared studies of model polar stratospheric cloud surfaces: Growth and evaporation of ice and nitric acid/ice, J. Geophys. Res. Atmospheres, 95(D13), 22423-22431, doi:10.1029/JD095iD13p22423, 1990.

Varilly, P. and Chandler, D.: Water evaporation: A transition path sampling study, J. Phys. Chem. B, 117(5), 1419-1428, 2013.

Vehkamäki, H. and Riipinen, I.: Thermodynamics and kinetics of atmospheric aerosol particle formation and growth, Chem. Soc. Rev., 41(15), 5160-5173, doi:10.1039/C2CS00002D, 2012.

Wikfeldt, K. T., Huang, C., Nilsson, A. and Pettersson, L. G. M.: Enhanced small-angle scattering connected to the Widom line in simulations of supercooled water, J. Chem. Phys., 134(21), 214506, doi:10.1063/1.3594545, 2011.

Willard, A. P. and Chandler, D.: Instantaneous liquid interfaces, J. Phys. Chem. B, 114(5), 1954-1958, 2010.

Winkler, P. M., Vrtala, A., Wagner, P. E., Kulmala, M., Lehtinen, K. E. J. and Vesala, T.: Mass and Thermal Accommodation during Gas-Liquid Condensation of Water, Phys. Rev. Lett., 93(7), 075701, doi:10.1103/PhysRevLett.93.075701, 2004.

Winkler, P. M., Vrtala, A., Rudolf, R., Wagner, P. E., Riipinen, I., Vesala, T., Lehtinen, K. E. J., Viisanen, Y. and Kulmala, M.: Condensation of water vapor: Experimental determination of mass and thermal accommodation coefficients, J. Geophys. Res. Atmospheres, 111(D19), doi:10.1029/2006JD007194, 2006.

Xu, Y., Petrik, N. G., Smith, R. S., Kay, B. D. and Kimmel, G. A.: Growth rate of crystalline ice and the diffusivity of supercooled water from 126 to 262 K, Proc. Natl. Acad. Sci., 113(52), 14921-14925, doi:10.1073/pnas.1611395114, 2016. 